\title{
ESTADO DEL ARTE DE IPTV Y CONSIDERACIONES TÉCNICAS PARA SU MIGRACIÓN A IPV6 EN COLOMBIA
}

José I. Torres R.

Estudiante de Ingeniería Electrónica en la Universidad Distrital "Francisco José de Caldas".

lastorresrojas@hotmail.com

Ramón E. Ramírez G.

Estudiante de Ingeniería Electrónica en la Universidad Distrital "Francisco José de Caldas".

ramonrg89@hotmail.com

\section{Danilo A. López S.}

Ingeniero Electrónico, MSc. en Teleinformática, docente de planta de la Universidad Distrital "Francisco José de Caldas", actualmente es Coordinador de la Maestría en Ciencias de la Información y las Comunicaciones de la misma Universidad.

dalopezs@udistrital.edu.co

Tipo: Artículo de revisión

Fecha de Recepción: Marzo 10 de 2011 Fecha de Aceptación: Mayo 10 de 2011

\section{STATE-OF-THE-ART IPTV AND TECHNICAL CONSIDERA- TIONS FOR IPV6 MIGRATION IN COLOMBIA}

\begin{abstract}
The main goals of this paper are to provide a comprehensive survey of state-of-the-art IPTV (Internet Protocol Television), and also to make suggestions for the implementation of this technology over an IPv6 network in Colombia. The convergence of telecommunications technologies has given rise to IP applications that allow access to digitized audiovisual content over networks using that protocol. Studies addressing the fundamental characteristics of television over data network such as performance, architecture, protocols, quality of service policies are presented. Some technical considerations for the IPTV proposal in Colombia are included at the end of this document.
\end{abstract}

Keywords: Internet Protocol Television (IPTV), bandwith, quality of Service, protocol, MPEG (Moving Picture Expert Group), set Top Box (STB).

\section{Resumen}

El artículo tiene como objetivos centrales hacer una revisión del estado del arte de IPTV (Televisión a través de IP) y una propuesta para la implementación de esta tecnología en Colombia sobre una red IPv6. La convergencia de las telecomunicaciones ha dado origen a aplicaciones IP que permiten acceso a contenidos audiovisuales digitalizados a través de redes que hacen uso de dicho protocolo. Se abordan estudios actuales que tratan características fundamentales de televisión sobre la red de datos, como el funcionamiento, la arquitectura, los estándares en los que se basa, propuestas para evaluar las políticas de calidad de servicio y por ultimo consideraciones técnicas para su implementación en una red IPv6.

Palabras clave: Televisión sobre el Protocolo de Internet (IPTV), ancho de Banda, calidad de servicio (Qos), MPEG (Grupo experto de imágenes en movimiento), decodificadores (STB). 


\section{INTRODUCCIÓN}

El actual avance de las telecomunicaciones ha permitido la utilización e implementación de nuevas aplicaciones para los usuarios, lo que impulsa un desarrollo en el mercado para ofrecer mejores experiencias al consumidor; gracias a esto surge el Internet Protocol Television desde este momento IPTV, este es uno de los avances con mayor auge en la actualidad por su gran variedad de utilidades, dentro de los que se encuentran "Triple play", Pago Por Ver (PPV), televisión móvil, grabador de video personal (PVR), Video Sobre Demanda (VoD), Guía de ProgramaciónElectrónica (EPG), entre otros [10]. La función interactiva que le presta al usuario, transformando este servicio de difusión broadcast en un solo sentido a una distribución multicast o unicast, donde las redes IP permiten interacción bidireccional entre el usuario y el proveedor del servicio [11], permiten que el cliente tenga el control sobre lo que desea ver y cuente con funcionalidades como pausar, grabar, retroceder y adelantar el contenido, además de permitir adicionar funciones de red como correo electrónico, navegación, datos, voz e intercambio de información.

Técnicamente, el proveedor hace uso de una conexión usando nodos que permiten encaminar cada paquete según la dirección de destino. Sobre esta base, la arquitectura IPTV está soportada en una plataforma de transmisión IP que llega a un decodificador (STB) [12], para que la señal pueda ser visualizada en un equipo terminal, sea este un televisor, un computador o incluso un equipo móvil celular [1].

En ocasiones es difícil ofrecer una calidad aceptable al usuario final, debido a los altos requisitos de anchode banda, retardo, disponibilidad, etc. Además, las características de estas arquitecturas, donde los nodos se conectan a través de muy diversas tecnologías, con enlaces asimétricos de subida y bajada de datos, con una altavariabilidad, saliendo y entrando del sistema en cualquier momento, hacen que no sea sencillo desplegar las funciones que posee el sistema [2]. Por este motivo es necesario la implementación de políticas de calidad de servicio (QoS) que ofrezcan una experiencia satisfactoria al subscriptor y cumplan con las necesidades de este [13].

Para la implementación en Colombia se hace evidente superar algunos aspecto de diseño, con el fin delograr masificación $y$ cubrimiento a nivel nacional, como son acceso a una conexión de banda ancha, la implementación de arquitecturas (ASON, DWDM,GMPLS, MPLS, SONET, P2P, etc.) [14], y una entidad regulatoria que permita la evolución, convergencia y escalabilidad. [15].

\section{FUNCIONAMIENTO DE IPTV}

La definición oficial aprobada por el grupo de la UIT es que: "IPTV es un servicio multimedia como la televisión, video, audio, texto, graficas y envío de datos sobre redes basadas en administración IP que proveen el nivel requerido de calidad de servicio, experiencia, seguridad, interactividad $\mathrm{y}$ confiabilidad" [16].

Existen topologías que inicialmente surgieron como redes de datos pero que evolucionaron hasta el punto de soportar nuevos contenidos y aplicaciones gracias al uso de protocolos abiertos, que facilitaron su rápida expansión [3]. Elavance y mejoramiento de las capacidades, calidades y niveles de servicio de Internet, han permitido gradualmente que puedan transmitirse satisfactoriamente contenidos que originalmente son sensibles al retardo y a la alteración del orden en que llegan los paquetes, por ejemplo, voz o imágenes en movimiento en tiempo real. IPTV se ha convertido hoy por hoy en la denominación más común para los sistemas de distribución por suscripción de señales de televisión y/o video [17]; se suministra junto con la conexión a Internet, proporcionado por un operador 
de banda ancha sobre la misma infraestructura, pero con un ancho de banda reservado para tal propósito. Se requiere un mínimo de $4 \mathrm{Mbps}$ para poder recibir la señal de TV comprimida según el formato MPEG-2 o MPEG-4; esto se consigue fácilmente con los nuevos estándares ADSL, como son ADSL2 y ADSL2+, que pueden llegar hasta 20 Mbps en bajada [18], VDSL con alcance de 65 Mbps de bajada y 15 Mbps de subida y sistemas de acceso óptico xPON con capacidades de 1 a 10 Gbps de bajada y 0.1 a 2.5 Gbps de subida [19]. La capacidad estimada para este nuevo servicio sobre IP se asume entre 1 y $2.1 \mathrm{Mbps}$ por cada canal de definición estándar (SDTV) y 8 Mbps por cada canal de alta definición (HDTV). Para dos canales simultáneos el espectro bruto resultante es de 2 a 4 Mbps para un servicio básico o bien 8 a $10.1 \mathrm{Mbps}$ si se incluye un canal HDTV, en ambos casos utilizando MPEG-4 para la codificación/compresión de la señal de video. A esta capacidad habría que añadirle el rango de frecuencias contratado para la conexión a Internet, que por bajo que sea ya implica la necesidad de manejar tasas de acceso de muy alta capacidad [4].

Entre los grupos más destacados de estandarización, está el DVB-IPI (DVB sobre infraestructura IP) [20], que es independiente del nivel físico, ya sea xDSL, cable, FTTH, xPON u otros. La base sobre la que se apoya DVB-IP es MPEG-2 y 4 para la compresión, el protocolo IGMP para la TV en directo y RTSP para video bajo demanda, entre otros [5].

En el caso de Colombia las redes no superan los 10 Mbps (en el 70\% de los casos) dada la distancia de los bucles de abonado, un acceso a varios Mbps de conexión a Internet sería adecuado para soportar este servicio.

\section{SERVICIOS Y APLICACIONES}

En la actualidad se encuentran en desarrollo aplicaciones que permiten múltiple $\mathrm{y}$ variada interactividad al usuario. Dependiendo del tipo de subscrición es posible acceder a diferentes servicios. Comúnmentese utilizan las siguientes:

Triple Play: Consiste en brindar por parte de un único operador prestaciones de voz, acceso a Internet y televisión para abonados, constituyendo un paquete básico [22] En Colombia se están mejorando las tecnologías de acceso y distribución local, con el fin de estaral nivel del mercado global en este tipo de aplicaciones.

Televisión Móvil: Permite descargar y guardar el contenido audiovisual en un formato portátil que se almacena en un espacio de disco duro. Este trabajo es realizado por el receptor STB, que también se encarga de transferir el archivo [10].

Grabador de Video Personal (PVR): Físicamente esta compuesto por una memoria no volátil con gran capacidad de almacenamiento y un software para el control y configuración de los contenidos, como aplicaciones de búsqueda avanzada.

Video sobre demanda (VoD): En este caso el flujo está dirigido a usuarios individuales y cada uno accede a un tipo específico de video; dentro de esta modalidad el contenido se difunde en modo unidifusión o Unicast [21].

Pago Por Ver (PPV): Es conocido como televisión a la carta, permite acceso a contenidos multimedia realizando un pago específico. A diferencia de $\mathrm{VoD}$, la señal se transmite de forma simultánea a todos los compradores [10].

Juegos: El crecimiento de la industria en este campo es amplio y tiene un futuro muy promisorio, debido a que se integra en forma bastante natural a la arquitectura IPTV, por lo que tales plataformas pueden incluir juegos para la TV. Aunque por ahora los que se ha implementado son desarrollos sencillos, la potencialidad que existe es llamativa 
y los fabricantes están trabajando para disponer de STBs que funcionen como consolas de juegos [22].

Adicionalmente se encuentran en desarrollo aplicaciones, entre estos el E-Learning que desarrolla una serie de cursos que fomentan el aprendizaje a diferentes grupos.

\section{FORMATOS Y PROTOCOLOS}

\subsection{Formatos de compresión de video}

La gestión de IPTV en la practica busca la optimización del tiempo de respuesta de la red, para lograr esto es necesario utilizar la compresión de video, ello implica que se cambie el formato; entre mayor sea la reducción, menor será el ancho de banda necesario para su envió y por ende su transmisión más rápida.

El estándar de compresión utilizado en IPTV es MPEG, que especifica como debe ser la trama de transporte de los datos. Hoy en día los formatos más utilizados son MPEG-2, MPEG-4 y H.200 de la serie ITU.

MPEG-2: Su aplicación inicial fue en la TV estándar (DTV) y de alta definición (HDTV). Está diseñado para trabajar con velocidades desde 2 hasta 100 Mbps. Es usado en reproductores DVD permitiendo imagen a pantalla completa más la ventaja de tener varios canales de audio.

MPEG-4: El objetivo fundamental es conseguir la mayor calidad de imagen posible. Los codificadores que se destacan son DivX y XviD, estos obtienen el mejor desempeño a velocidades bajas de transmisión. Puede ser utilizado en dispositivos móviles que soporten $3 \mathrm{G}$ y cámaras digitales.

H.263: Surgió al mismo tiempo que MPEG4 parte 2 (con el cual es compatible). La idea fundamental de este algoritmo fue proporcionar mejor apreciación audiovisual para video telefonía y video conferencia.
H.264: Define un codec de alta compresión (es el mismo MPEG-4 parte 10). Puede alcanzar la máxima calidad de MPEG-2 pero con la mitad de los recursos y sin aumentar la complejidad del diseño. Tiene una sintaxis sencilla que mejora la integración con diferentes protocolos [7].

La Unión Internacional de Telecomunicaciones (ITU-T) estandarizó en 2008 las tecnologías de compresión de video avanzadas H.264/MPEG4, que presentan una mejora sustancial en cuanto al rendimiento de MPEG2, que soportan condiciones heterogéneas de transporte, especialmente para contenidos de VoD y televisión de alta definición (HDTV) [23].

\subsection{Protocolos de transmisión}

Los estándares utilizados para el servicio de IPTV, son los de transporte, UDP y RTP / RTCP, los de control de sesión, como RTSP y SDP, y el protocolo de internet. Un servicio de IPTV típico usa un esquema de empaquetamiento de tipo MPEG2-TS/RTP/UDP/ IP.

\subsubsection{UDP}

Descrito en la RFC768. En IPTV se utiliza para dar servicio no orientado a la conexión y no fiable. Permite el envió de datagramas a través de la red sin que haya establecido previamente la conexión. Su uso principal es dar soporte a DHCP, BOOTP, DNS, SNMP; así como para las trasmisiones de audio y video en tiempo real, donde no es posible utilizar TCP por las demoras (retardos) en que se incurrirían.

\subsubsection{RTP}

Estandarizado en el RFC 3550. Es el protocolo de trasporte para flujos multimedia en Internet. IPTV lo utiliza para proporcionar información temporal y de sincronización de los flujos multimedia.

\subsubsection{RTCP}

Definido en la RFC 3605. El Protocolo de Control en Tiempo Real, se basa en la trans- 
misión periódica de paquetes de control a todos los participantes dentro de una sesión. En IPTV reserva recursos en la red para la transmisión en tiempo real.

\subsubsection{SDP}

Protocolo de descripción de sesión SDP, definido en la RFC 2327, es un protocolo de control de la capa de aplicación que ofrece toda la información antes de iniciar una sesión. Solo proporciona un formato para la descripción de la sesión, sin incorporar ningún protocolo de transporte.

\subsubsection{RTSP}

Protocolo de Transmisión en Tiempo Real, definido en la RFC 2326. IPTV lo utiliza para establecer y controlar uno o muchos flujos sincronizados de datos, ya sean de audio o de vídeo. Le permite a un cliente realizar operaciones de control remoto sobre un servidor de streaming. Cabe dejar claro que RTSP no es un estandar para enviar información; para ello, se suele utilizar RTP [7].

Para las transmisiones multicast son necesarios estándares especiales que se encarguen de la difusión a diferentes grupos de terminales que pertenecen a la red, el más utilizado para IPTV es IGMP.

\subsubsection{IGMP}

Utilizado para intercambiar información concerniente al estado de pertenencia entre enrutadores IP y miembros de grupo de multidifusión, existen tres versiones; la primera descrita en la RFC 1112, que define procesos de consulta-respuesta, adherencia, desincorporación a grupos y un mecanismo de supresión de reporte [24], la segunda definida en la RFC 2236, maneja el mismo tipo de mensaje de consulta y de reporte que la anterior diferenciándose por dos excepciones: 1) los mensajes de consulta se dividen en dos subtipos: generales y específicos [25], 2) utiliza un proceso de simple elección para elegir el consultor multicast. Por último, la tercera versiónespecificada en la RFC 3376, amplía los meca- nismos de adherencia y desincorporación de anfitriones [26]. Permite a los miembros de un grupo seleccionar las fuentes específicas de las cuales desean recibir tráfico aún dentro del mismo grupo [27].

\section{TECNOLOGIAS DE DISTRIBUCION DE REDES IPTV}

La transmisión de televisión sobre IP utiliza diferentes tipos de distribuciones de red, que determinan muchos factores, como la rata de transferencia, arquitecturas de transporte, etc. La Fig. 1 está basada en el reporte técnico TR-126 del DSL Forum que describe la arquitectura de un estructura IPTV, la grafica muestra las diferentes funciones de los dispositivos de la red.

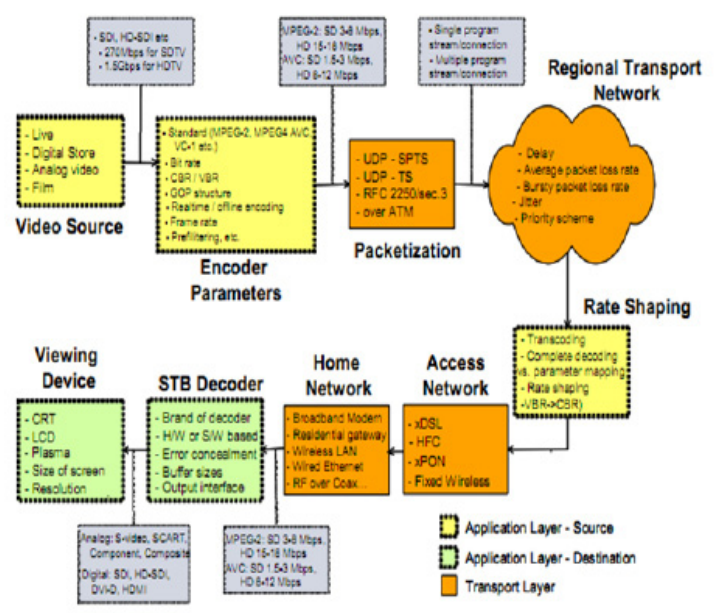

Fig. 1. Diagrama de bloques de una red IPTV punto a punto [6].

Dentro de las principales tecnologías ofertadas a nivel de distribución están:

\subsection{IPTV sobre entornos ópticos}

Las redes ópticas pasivas (PON) son punto a multipunto y estan soportadas sobre componentes ópticos entre un nodo central denominado OLT (Terminal óptico de línea) y los equipos en campo ONT (Terminal óptico de red) [29], en cualquiera de sus configuraciones como fibra hasta el hogar (FTTH), fibra hasta el edificio (FTTB) ofibra 
hasta el nodo (FTTN).

PON es una técnica a la que están convergiendo actualmente la mayoría de sistemas IPTV gracias a las ventajas en cuanto a ancho de banda, mejor desempeño frente al ruido y velocidad de transmisión, además de requerir únicamente equipos activos en los extremos y divisores ópticos pasivos para guiar el tráfico intermedio y repartir la señal por las fibras que se dirigen a cada punto de conexión [29].

Existen diferentes variantes para PON que dependen del tipo de arquitectura de transporte:

\subsubsection{APON}

Utiliza el estándar ATM, la velocidad de operación depende de si la conexión es simétrica o asimétrica. En el primer caso opera a velocidades alrededor de $155 \mathrm{Mbps}$ y para el segundo alcanza capacidades de envío en bajada de 155.52 a 622.08 Mbps y subida de 155.52 Mbps [30].

\subsubsection{BPON}

Broadband-PON se define en varias revisiones al estándar ITU-T 983 que incluyen desde la G.983.1 que es la original hasta la G.983. Surgió como una mejora a APON para integrar y obtener acceso a más servicios como Ethernet, distribución de video, VPL, y multiplexación por longitud de onda (WDM) logrando un mayor espectro de frecuencias.

La especificación G.983.1 de B-PON define una arquitectura de forma simétrica, a una velocidad de 155 Mbps. Esta norma fue revisada en el año 2003 para lograr un aumento en la rata de transferencia y para permitir formas asimétricas con $155 \mathrm{Mbps}$ de subida y 622 Mbps de bajada [31].

\subsubsection{EPON}

Estandarizado como PON sobre Ethernet con una tasa promedio de $1 \mathrm{Gbps}$ en modo simétrico [32].

\subsubsection{GPON}

En esta tecnología de última milla, se asigna una longitud de onda para el tráfico de datos (Internet, VoIP, IPTV, etc.) downstream $(1.490 \mathrm{~nm})$ y otra para el tráficoupstream $(1.310 \mathrm{~nm})$. Además, a través del uso de WDM (multiplexación por división de onda), se asigna una tercera longitud de $1.550 \mathrm{~nm}$ que está dedicada para el broadcast de vídeo RF(analógico, digital, HDTV). De este modo, el vídeo/TV puede ser ofrecido mediante dos métodos distintos simultáneamente: RF (radio frecuencia) e IPTV. Mediante RF las operadoras de cable pueden hacer una migración gradual hacia la televisión IP. En este caso, las ONT dispondrán de una salida para vídeo RF coaxial que irá conectada al STB tradicional. Con IPTV la señal, que es transformada por la cabecera en una cadena de datos IP se transmite sobre el mismo enlace como datos para acceso a Internet de banda ancha. El STB conectado mediante Gigabit Ethernet al ONT, convertirá de nuevo la cadena de paquetes en una señal entendible para el receptor. Mediante IPTV y GPON, cuyos equipos incorporan capacidades de QoS y multicast IP avanzados, los operadores pueden ofrecer varios canales de alta calidad de imagen y sonido, incluidos HDTV, así como proporcionar prestaciones interactivas y personalizadas, características que no factibles con vídeo RF [33].

En la tabla 1 se observa que existe una clara ventaja para una determinada red FTTB (fibra hasta el edificio) basada en GPON sobre una red basada en EPON, debido a la mayor razón de división, a la rata de envío, a la eficiencia ya la capacidad de canal, lo que resulta en la reducción de OLTs con respecto a EPON [34] y mayor uso por parte de los ISPs. 
Tabla 1. Diferencias entre tecnologías de distribución PON. [32].

\begin{tabular}{|c|c|c|c|}
\hline \multicolumn{4}{|c|}{ Diferencias BPON, GPON, GEPON } \\
\hline $\begin{array}{c}\text { Organiza- } \\
\text { ción }\end{array}$ & $\begin{array}{c}\text { BPON } \\
\text { ITU- } \\
\text { T/FSAN }\end{array}$ & ITU-T/FSAN & IEEE EFM \\
\hline Standard & G.983 & G.984 & IEEE 802.3 \\
\hline $\begin{array}{c}\text { Downstre- } \\
\text { am }\end{array}$ & $1,25 \mathrm{Gbps}$ & $2,5 \mathrm{Gbps}$ & $1 \mathrm{Gbps}$ \\
\hline Upstream & $622 \mathrm{Mbps}$ & $2,5 \mathrm{Gbps}$ & $1 \mathrm{Gbps}$ \\
\hline $\begin{array}{c}\text { Max Split } \\
\text { Ratio }\end{array}$ & $1: 32$ & $1: 128$ & $1: 32$ \\
\hline $\begin{array}{c}\text { Payload } \\
\text { ATM }\end{array}$ & $\begin{array}{c}\text { ATM, TDM,GE, } \\
\text { MPLS,GMPS, } \\
\text { ASON }\end{array}$ & GE \\
\hline $\begin{array}{c}\text { Interfaz de } \\
\text { red }\end{array}$ & $\begin{array}{c}\text { ATM, } \\
\text { TDM,GE }\end{array}$ & $\begin{array}{c}\text { ATM, TDM,GE, } \\
\text { MPLS,GMPS, } \\
\text { ASON }\end{array}$ & GE \\
\hline $\begin{array}{c}\text { Interfaz de } \\
\text { Acceso }\end{array}$ & $\begin{array}{c}\text { ATM, } \\
\text { TDM, } \\
10 / 100\end{array}$ & $\begin{array}{c}\text { ATM, TDM, } \\
10 / 100\end{array}$ & $10 / 100$ \\
\hline Distancia & $20 \mathrm{Kms}$ & 20 Kms & $20 \mathrm{Kms}$ \\
\hline
\end{tabular}

\section{$5.2 \quad$ IPTV sobre $x D L S$}

Garantizan el soporte de flujos digitales a altas velocidades sobre las líneas telefónicas existentes en los hogares y los negocios. Ofrecen conexiones que van desde los 100 Kbps hasta los 52 Mbps (descendentes). Pero su aplicación en un punto determinado está limitada por factores como la distancia entre la central local y la vivienda, el calibre del cable y el tipo de DSL.

\subsubsection{ADSL}

De característica asimétrica, donde el ancho de banda de bajada es superior al de subida, alcanzando los $10 \mathrm{Mbps}$ en un sentido y $1 \mathrm{Mbps}$ en el otro. Es adecuado para el uso residencial ya que habilita a los usuarios para llamadas telefónicas y navegación de forma simultánea.

\subsubsection{VDSL}

Es la modalidad más rápida de xDSL ya que puede alcanzar velocidades entre 13 y 52 Mbps desde la central hasta el abonado y de 1,5 a 2,3 Mbps en sentido inverso. La máxima distancia que puede existir entre los dos módems VDSL no puede superar los 1.371 metros.

\subsubsection{HDSL}

Es simétrica. Las conexiones HDSL son apropiadas para servidores (web, FTP, ficheros) y otros usos comerciales, como la video conferencia. Permite tasas superiores a los $2.3 \mathrm{Mbps}$, incluyendo un segundo par de cobre, lo que implica alcances de 4.6 Mbps a una distancia máxima de $3 \mathrm{Km}$.

\subsubsection{ADSL2 y ADSL2+}

Son actualmente las conexiones más ampliamente utilizadas en el mundo, ya que han mejorado las características de rapidez, alcance y consumo energético de la versión ADSL original. ADSL2 oscila entre los 8 y 12 Mbps extendiendo el alcance del ADSL original a unos 300 metros. ADSL2+ ofrece interconexiones de hasta $16 \mathrm{Mbps}$ a una distancia de unos $1.5 \mathrm{Km}$ [35].

\subsection{IPTV sobre entornos satelitales}

Los enlaces satelitales pueden proveer mayores rangos de espectro a diferencia de otras redes de transmisión terrestre, en la actualidad son muy usadas para servicios Triple Play. Algunos ISPs satelitales basan sus plataformas para envío de contenidos audiovisuales por IP. La infraestructura usada para soportar estos mecanismos de distribución IPTV esta mostrada en la Fig. 2 , donde se observa que el eje de converencia es el satélite.

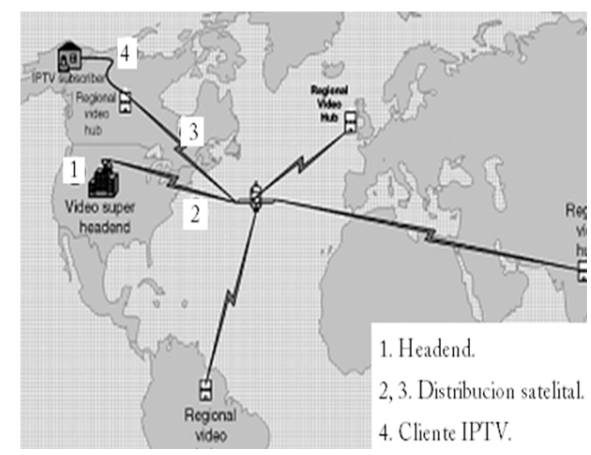

Fig. 2. IPTV sobre una red satelital [28].

Además de las ventajas de esta topología, como la adquisición de fuentes, hay que te- 
ner en cuenta que puede ser muy costoso debido a los elevados precios de arrendamiento de frecuencias.

\subsection{IPTV sobre entornos inalámbricos}

Los avances en cuanto a capacidad de ancho de banda y acceso a redes inalámbricas hacen que este tipo de distribución sea una tendencia actual entre los operadores de televisión sobre IP, porque se máxima el número de subscriptores [23].

Los entornos geográficos combinados con otros factores como especificaciones de equipo y condiciones ambientales pueden tener un impacto entre el cliente IPTV y la estación base que provee la señal inalámbrica. Wimax posee una transferencia teórica de $60 \mathrm{Mbps}$ con un área de cobertura de 6 a $10 \mathrm{Km}$. dependiendo del hardware utilizado. Un esquema común es mostrado en la Fig. 3.

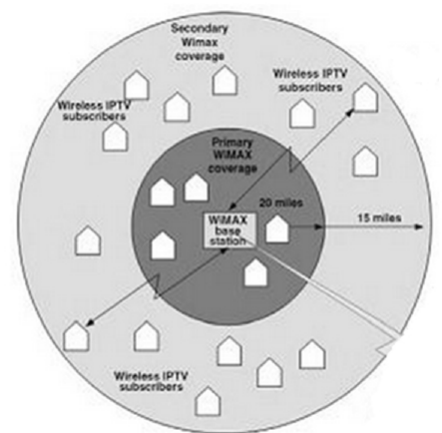

Fig. 3. Diagrama simplificado de transporte de trafico IPTV en una red inalámbrica[28].

\section{ARQUITECTURAS DE TRANSPORTE}

En las redes IPTV se plantean dos distribuciones diferentes, la primera de ellas es similar a la televisión por cable, que es di-fusión por broadcast; un ejemplo de este son los canales de noticas, que operan en vivo. La segunda se realiza por el equipo terminal del usuario, donde el cliente accede a un determinado programa televisivo.

\subsection{Elementos de un sistema de IPTV}

Una red IPTV de alcance metropolitano esta conformada por algunos componentes básicos, los cuales se observan en la Fig. 13, y son descritos a continuación.

Cabecera o Headend: Incluye las funciones para recibir las señales en vivo (transmitidas vía satélite o provistas por fuentes locales) y convertirlas al formato necesario para su difusión y posterior recepción por los STB. Está constituido por: antenas para recepción de contenido audiovisual, receptores, servidores de codificación/transcodificación, el sistema de ajuste de ancho de banda del flujo de información (rate shaping), y equipos de encaminamiento que conforman los streams a insertar en la red de un operador.

El Sistema de video y audio a demanda: Almacena y transmite las señales de video $\mathrm{y}$ audio que pueden ser solicitadas por los usuarios. El sistema de VoD también se encarga de ofrecer los servicios de PVR y Time Shift TV (TSTV) cuando estos se implementan en la red (y no en el hogar del usuario con un disco duro local).

El sistema de control de derechos y control de acceso: Encripta la información de modo que no se vulneren los derechos de propiedad intelectual al ser transmitidos.

Plataforma de gestión de aplicaciones o Middleware: Define y coordina la forma en que el usuario interactúa con el servicio de TV por la red de datos, y soporta la interacción de los distintos servidores del sistema. Constituye un tipo de servidor de portal que es accedido y utilizado por una aplicación "cliente" que se ejecuta en el STB, pero además de la presentación de los servicio al cliente, tiene la función de coordinar los subsistemas de la arquitectura IPTV. Por ejemplo ofrece en el menú del usuario un video para ser descargado (la lista de videos disponibles es coordinada por operadores de la red y obtenida del sistema de VoD), si el cliente decide comprarlo entonces realiza el débito y le avisa al sistema que el usuario tiene permiso para descargarlo. 
Codificadores o Set Top Box (STB): Son los equipos que se conectarán con el TV del cliente. Deben decodificar las señales de video (que se transporta como flujo de información multimedia sobre IP) para hacerlas compatibles con un televisor. Cada STB dispone de un control remoto utilizado por los subscriptores para enviar las órdenes al sistema.

Dispositivo de entrada: Deben disponer de al menos dos puertas Ethernet para mapear diferentes calidades de servicio. Un gateway es para conectar la red residencial de Internet y la otra para conectar a los STB, que requieren de QoS. Si el operador implementa cada servicio en una LAN virtual diferente, cada VLAN se mapeaen una puerta Ethernet distinta [6].

La Fig. 1 muestra de forma detallada los bloques que posee un sistema IPTV. Puntualmente video source y encoder parameters es lo que se conoce como Headend.

\section{CALIDAD DE SERVICIO}

Dentro de los parámetros de QoS para un sistema de TV sobre IP se puede encontrar la disponibilidad del canal, el tiempo de comienzo del visionado, fallos y retardos en el cambio del canal [36]. Para el tráfico de bajada, IPTV ofrece servicios diferenciados para el usuario, en los cuales, este, identifica los pa-quetes con una determinada etiqueta que marca la prioridad y el trato que deben recibir por parte de los nodos [37], El flujo de subida es monitorizado de forma que se pueda controlar el acceso para garantizar la QoS [38].

Las métricas que definen una señal clara y continua, incluyen el jitter, el número de paquetes fuera de orden, la probabilidad de pérdida de paquetes y errores en la red, el tiempo de unión multicast, el retardo, etc. Dentro de las variables para voz están el jitter, retardo, ratio de pérdida de paque- tes de voz, y MOS (Mean Opinion Score), un parámetro que refleja la opi-nión subjetiva del usuario [2].

\subsection{Recomendaciones de la ITU para QoS}

Consecuentemente con lo anterior, la ITU determina que el retardo para todos los servicios IPTV debe tener un umbral máximo de $200 \mathrm{~ms}$, mientras que para jitter se establece una variabilidad máxima de 50 ms. En lo referente a la tasa de pérdida de paquetes, se exige que para transmisiones de contenidos de video de alta definición utilizando códec MPEG-4, no se sobrepase una relación de 10 a 6 paquetes perdidos con respecto a paquetes transmitidos, ya que la pérdida depende de la tasa de transmisión de bits; finalmente se recomienda un retardo máximo de $2 \mathrm{~s}$ para zapping y $200 \mathrm{~ms}$ para las funciones TsT (control sobre el tiempo de reproducción del contenido) y EPG (guía electrónica de programación). Finalmente.se considera que los requerimientos de ancho de banda son el factor de mayor relevancia para los servicios IPTV, dado que la compresión y digitalización exigen altas tasas de transmisión de bits para que la pérdida de datos de información sea baja [8].

A continuación se presentan tres estudios acerca del rendimiento de los parámetros relacionados con QoS cuando se presta un servicio IPTV; cada uno de estos muestra las topologías que se abordaron en el análisis del comportamiento de la Televisión sobre IP para las diferentes variables analizadas.

Las variables analizadas en los estudios son perdida de paquetes, retardo en el cambio de canal y ancho de banda requerido ya que estas son las mas relevantes e importantes en cuanto a calidad de servicio y de experiencia para el usuario. 


\section{2 Análisis del rendimiento de la transmisión de IPTV sobre ADSL, WIFI y LAN Extended}

Este estudio fue desarrollado en la tesis de nombre "Análisis del rendimiento de la transmisión de IPTV sobre ADSL, WIFI y LAN Extended" realizado por C. Viloria, J. Freja, Y. Donoso en el año 2008 [39]. En este documento se analizan pruebas sobre distintas plataformas tecnológicas (ADSL, WIFI y LAN Extended), variando la tasa de transmisión utilizada, el códec de video implementado y la aplicación o no de la calidad de servicio (QoS); con el fin de observar como se ve afectada la pérdida de paquetes en el servicio de televisión sobre IP.

La plataforma LAN Extended es utilizada para extender el rango de las redes inalámbricas, proporcionando un camino para la transferencia de datos entre dos LAN.

\subsubsection{Implementación}

La implementación utilizada para la realización de las pruebas consta de un servidor que se encuentra del lado de la central, un computador receptor y un Set Top Box, que se encarga de entregar el flujo a un televisor. Como se puede observar en la Fig. 4. Al igual, del lado de los usuarios se coloca un equipo en el que se hace correr un software que monitorea y registra el flujo de paquetes para obtener los datos.

Por último, se añade un equipo transmisor de datos del lado del servidor para generar flujo FTP en la red, lo que permite el intercambio de datos entre receptor y transmisor.

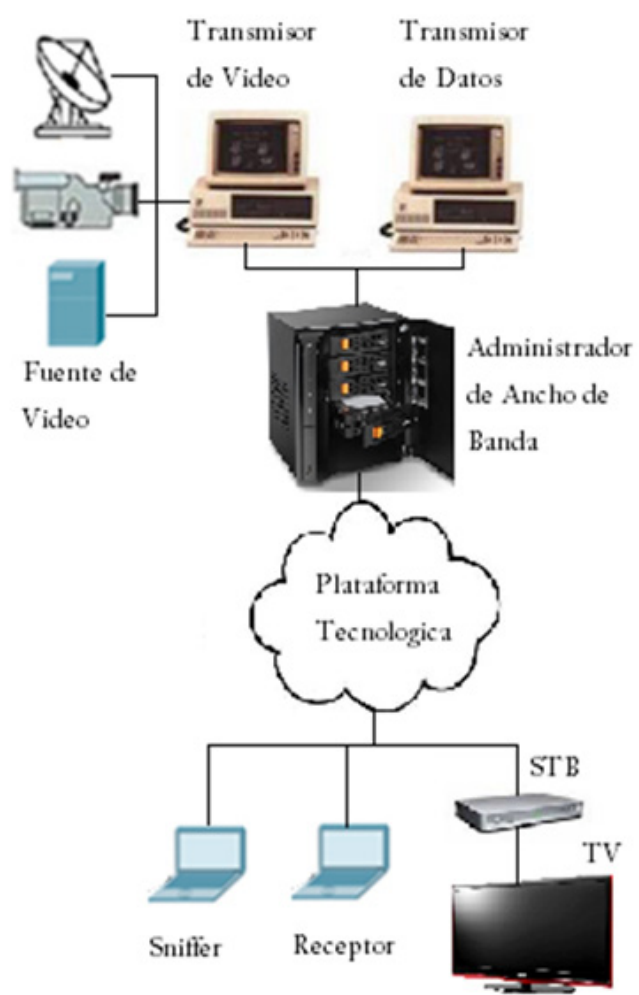

Fig. 4. Arquitectura general del sistema para IPTV.

\subsubsection{Resultados}

Los resultados obtenidos para las diferentes tecnologías sobre las cuales se implementó IPTV en las pruebas, se presentan a continuación:

\section{- ADSL}

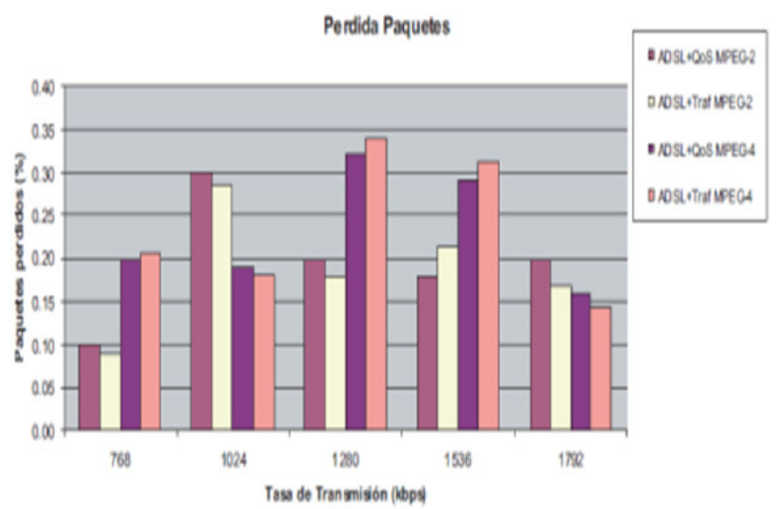

Fig. 5. Resultados de pérdida de paquetes en pruebas de IPTV sobre ADSL [39].

En la Fig. 5 se observa que la pérdida de paquetes es ligeramente más baja con códec 
MPEG2 cuando hay tráfico o se implementa QoS y que ADSL es indiferente al códec que se utiliza o a la posibilidad de implementación de QoS ya que se comporta de una manera muy similar en todos los casos.

\section{- WIFI}

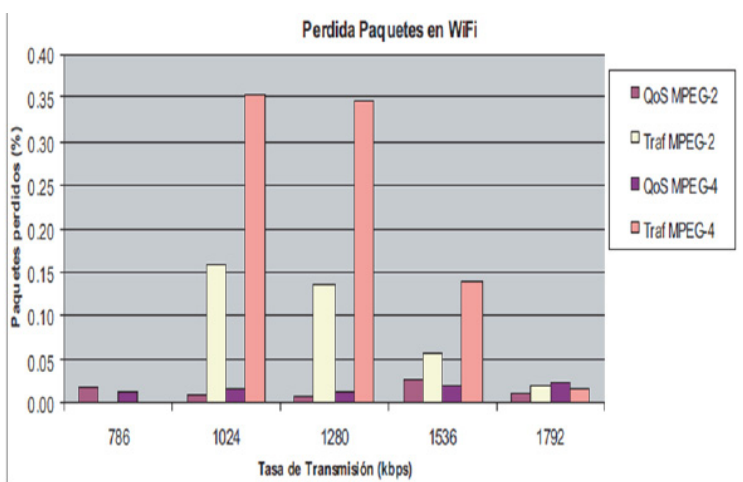

Fig. 6. Resultados de pérdida de paquetes en pruebas de IPTV sobre WIFI[39].

En la Fig. 6, se muestran los resultados de las pérdidas de paquetes en WIFI, se concluye que la diferencia es grande cuando se aplica calidad de servicio; si se utiliza códec MPGE2, se obtiene un mejor comportamiento del servicio, lo cual es notorio en las pruebas sin QoS; respecto a la implementación con QoS, no se evidencia gran diferencia entre los códec utilizados.

\section{- LAN Extended}

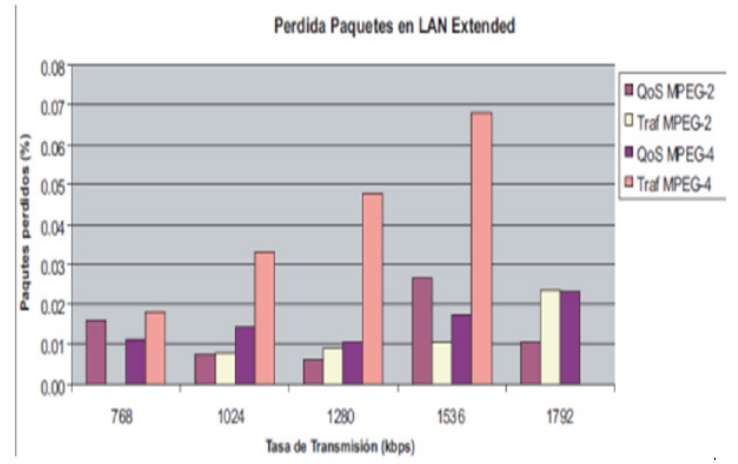

Fig. 7. Resultados de pérdida de paquetes en pruebas de IPTV sobre LAN extended[39].
En la Fig. 7 se evidencia que cuando se aplica QoS, la pérdida de paquetes tiene un mejor comportamiento. Además, el desempeño de este parámetro del servicio respecto al codec es similar en tasas bajas pero a medida que se aumenta, su comportamiento varía.

En la actualidad, se han presentado varias propuestas para disminuir la pérdida de información, tales como: la reducción en el tiempo de implementación del STB, optimización en el diseño de la red, decodificación y desencriptación de datos, los cuales representan el mayor porcentaje de las pérdidas [39].

\subsubsection{Reducción del retardo en el cam- bio de canal en servicios IPTV}

A continuación se presenta un estudio acerca de la reducción del retardo que se presenta en la red cuando un usuario cambia de canal (zapping); esto con el fin de mejorar la calidad de experiencia del servicio en el usuario, dado que es molesto para el cliente esperar cuando se esta cambiando de canal. La ITU establece un máximo de dos segundos para este tipo de retardo, tiempo que es bastante amplio e incomodo para el subscriptor.

El estudio es adaptado del articulo, que tiene como nombre "Reducción del retardo en el cambio de canal en servicios IPTV", realizado por F. Moumtadi, M. Escobar, R. López y S. Landeros. En Enero de 2008 [40].

El estudio esta basado en el método de grupos adyacentes, el cual reduce el tiempo de adquisición del nuevo canal haciendo el pedido no solamente de ese canal sino que también el de los adyacentes.

La arquitectura implementada para la realización se muestra a continuación. 


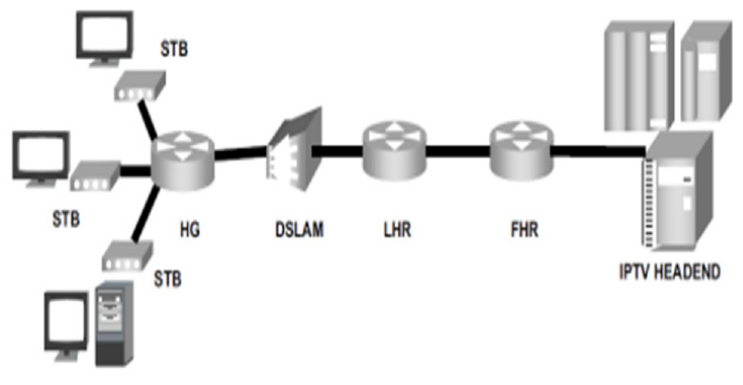

Fig. 8. Arquitectura general del sistema para IPTV[40].

Se asume que el ancho de banda de la red de acceso (entre el LHR y el HG), es lo suficientemente grande como para soportar diversos flujos multicast de manera simultánea, ante la solicitud por parte del STB de adquirir un nuevo canal mediante el envío de un mensaje de entrada al grupo, el HG podría no sólo enviar el mensaje de entrada correspondiente al grupo de ese canal, sino también para los grupos de los canales adyacentes.

Para el proceso de simulación, la cual fue desarrollada en Matlab, se tienen en cuenta las siguientes condiciones:

- El método de grupos adyacentes fue aplicado con dos canales adyacentes, uno hacia arriba y otro hacia abajo, y se analizó su influencia en el tiempo de cambio de canal que experimentan los usuarios.

- Se realizan diez cambios de canal entre los tres STB's del sistema. Se eligió un STB al azar en cada uno de los casos.

- Los cambios se efectuaron de dos formas distintas, hacia canales aleatorios y hacia canales adyacentes[40].

Se analizan cuatro escenarios diferentes:

A. Metodología de cambio tradicional con cambios hacia canales adyacentes.

B. Metodología de cambio mejorada con cambios hacia canales adyacentes.

C. Metodología de cambio tradicional con cambios hacia canales aleatorios.

D. Metodología de cambio mejorada con cambios hacia canales aleatorios.

Sobre cada escenario se hicieron variar tanto el número de usuarios como el de canales para analizar la influencia de estos en el tiempo de cambio y se obtuvieron los resultados que se presentan a continuación:

Para los escenarios 1 y 2 y tomando 100 canales y 50 usuarios, la reducción que se alcanzó con el método fue de $330 \mathrm{~ms}$ aproximadamente.

Para los escenarios 3 y 4 en los mismos 100 canales y 50 usuarios, se observa que el retardo se mantiene en un promedio de 380 ms; esta vez, con el método implementado se obtiene un retardo con un valor promedio aproximado de $200 \mathrm{~ms}$, logrando una reducción del tiempo de adquisición cerca de $180 \mathrm{~ms}$. Esta reducción del tiempo no es tan amplia como cuando se cambia a canales adyacentes, debido a que, con la disponibilidad de los canales adyacentes en el HG, el DSLAM o el LHR, los canales solicitados pueden o no estar disponibles en estos enrutadores.

El estudio arrojo dos resultados importantes que pueden ser resumidos en las siguientes graficas:

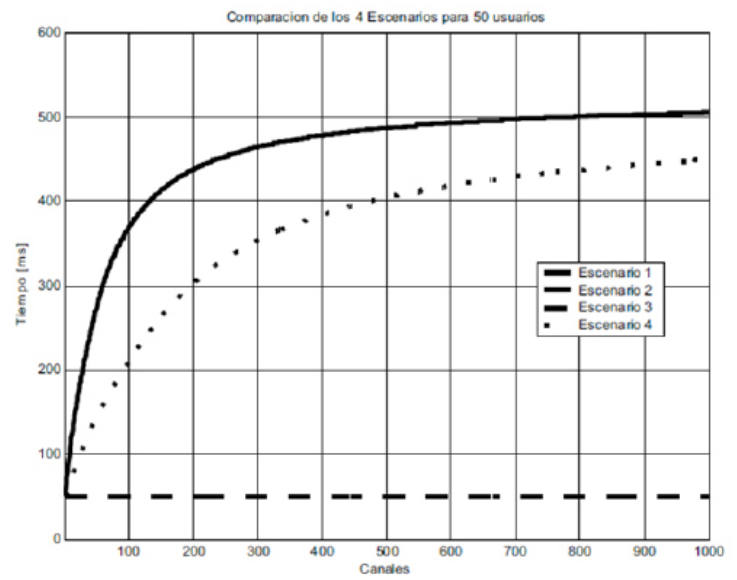

Fig. 9. Gráfica comparativa de los 4 escenarios, variando la cantidad de canales [40].

Como se puede observar en la Fig. 9, cuando 
se aumenta el número de canales se aprecia un crecimiento en el retardo debido a que es menos probable que el canal deseado esté siendo visto por algún otro usuario.

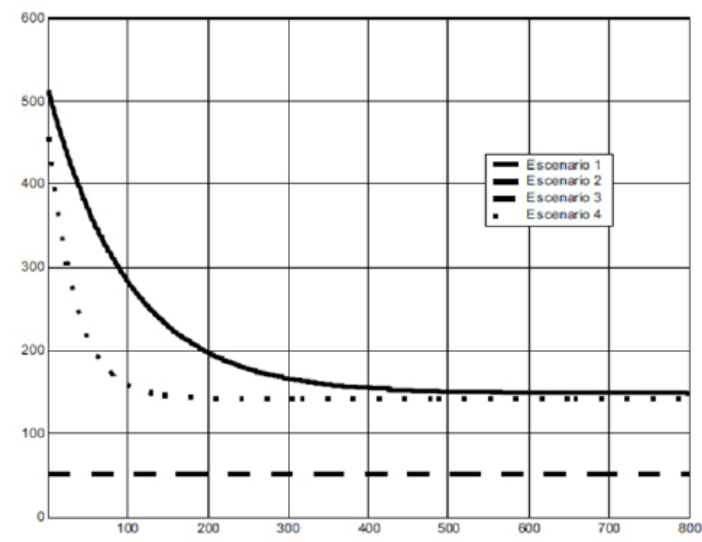

Fig. 10. Gráfica comparativa de los 4 escenarios, variando la cantidad de usuarios [40].

En la Fig. 9, se deduce que el retardo disminuye si aumenta el número de usuarios, ya que es más probable que el canal deseado esté siendo visto por algún otro subscriptor y por lo tanto se encontrará disponible más cerca del cliente que lo solicite.

Se concluye que al implementar el método de grupos adyacentes se obtiene una mejora considerable del retardo si se cambia a canales adyacentes; el retardo tendrá un valor constante igual al tiempo entre la transmisión del Joinmessage y la recepción del primer paquete del grupo multicast, correspondiente al nuevo canal enviado directamente del HG, por lo que el tiempo de adquisición será casi inmediato sin importar el número de usuarios. Sin embargo, el método sólo servirá si el ancho de banda de la red de acceso es suficientemente grande para soportar diversos flujos multicast, de lo contrario, simplemente el método afectaría a la calidad de experiencia del usuario en vez de beneficiarla [40].

\subsubsection{Análisis de requerimientos de an- cho de banda para servicios IPTV} Este estudio es adaptado del articulo, que tiene como nombre "Análisis de requerimientos de ancho de banda para servicios
IPTV" realizado por F. Mieles, S. Torres [8]. y pretende dimensionar la capacidad de canal mínima requerida para prestar el servicio de IPTV y así asegurar calidad del servicio.

Se considera una conexión en tiempo real que utiliza los servicios de VoD y televisión de difusión.

Video sobre demanda: Cada VSO (Oficina de Servicio de Video) ofrece el servicio de IPTV al $20 \%$ de cierto número de abonados de una ciudad. Cada uno de los usuarios cuenta con 2 televisores. Del total de TV solo el 20\% representa el pico máximo de tráfico, es decir, se dimensiona la red para el valor de mayor tráfico. Sí cada equipo generan una solicitud de servicios VoD, $90 \%$ en definición estándar a un flujo de bits de $2.1 \mathrm{Mbps}$ y el $10 \%$ restante a 10 Mbps, el BW requerido se puede calcular de la siguiente forma: Adaptado de [8].

Ancho de banda $=$ Num de $T V^{*}($ Cap.requerida $)$

La Ec. 1 calcula el ancho de banda total requerido por un enlace al multiplicar el número de televisores, por la capacidad de canal requerida por cada uno.

Televisión de difusión: El VHO (Video Hub Office: nodo encargado de agregar contenidos regionales ó locales y de VoD para un área geográfica que comparte preferencias en la parrilla de programación) envía el flujo de video de 100 canales, de los cuales, el $20 \%$ se transmite a una tasa de $10 \mathrm{Mbps}$ (alta definición) y el $80 \%$ restante a 2 Mbps (definición estándar), resultando en:

$$
\begin{gathered}
B W=100 *(0.8 * 2.1+0.2 * 10) \\
B W=368 \mathrm{Mbps}
\end{gathered}
$$

Se concluye que con un mínimo de aproximadamente $368 \mathrm{Mbps}$ se pueden transmitir 100 canales de televisión de difusión.

El límite mínimo para el dimensionamiento 
de la red metropolitana se obtiene sumando los requerimientos de capacidad de las ecuaciones (1) y (2).
En la tabla anterior se observan los datos que resultan al realizar el proceso descrito anteriormente con diferente número de abonados, es evidente que dado los valo-

Tabla 2. Dimensionamiento de ancho de banda total de una red metropolitana.

\begin{tabular}{|c|c|c|c|c|c|c|c|}
\hline \multirow{2}{*}{$\begin{array}{l}\text { Número } \\
\text { de } \\
\text { abonados }\end{array}$} & \multirow{2}{*}{$\begin{array}{l}\text { Clientes } \\
\text { IPTV } \\
(20 \%)\end{array}$} & \multirow{2}{*}{$\begin{array}{l}\text { Numero de } \\
\text { Televisores }\end{array}$} & \multirow{2}{*}{$\begin{array}{c}\text { Pico } \\
\text { máximo } \\
\text { de tráfico } \\
(20 \%) \\
\text { [TVs] }\end{array}$} & BW de Vod & $\begin{array}{c}\text { BW de TV } \\
\text { por difusión }\end{array}$ & \multirow[b]{2}{*}{ Total } & \multirow{2}{*}{$\begin{array}{c}\text { Ancho de } \\
\text { banda total } \\
\text { [Mbps] }\end{array}$} \\
\hline & & & & $\begin{array}{l}\text { SDTV (90\%) } \\
\text { HDTV }(10 \%)\end{array}$ & $\begin{array}{c}\text { SDTV }(80 \%) \\
\text { HDTV } \\
(20 \%)\end{array}$ & & \\
\hline 10000 & 2000 & 4000 & 800 & $2312 \mathrm{Mbps}$ & $368 \mathrm{Mbps}$ & $2680 \mathrm{Mbps}$ & $4466,66+$ res. \\
\hline 15000 & 3000 & 6000 & 1200 & $3468 \mathrm{Mbps}$ & $368 \mathrm{Mbps}$ & $3836 \mathrm{Mbps}$ & $6393,33+$ res. \\
\hline 20000 & 4000 & 8000 & 1600 & $4624 \mathrm{Mbps}$ & $368 \mathrm{Mbps}$ & 4992 Mbps & $8320+$ res. \\
\hline 25000 & 5000 & 10000 & 2000 & $5780 \mathrm{Mbps}$ & $368 \mathrm{Mbps}$ & $6148 \mathrm{Mbps}$ & 10246,6 + res. \\
\hline 30000 & 6000 & 12000 & 2400 & $6936 \mathrm{Mbps}$ & $368 \mathrm{Mbps}$ & $7304 \mathrm{Mbps}$ & 12173,3 + res. \\
\hline
\end{tabular}

res totales es necesaria una infraestructura compleja que permita suministrar de manera confiable estas magnitudes. Cabe aclarar que no es suficiente garantizar el mínimo requerimiento de capacidad, ya que además se debe tener en cuenta el aprovechamiento de los enlaces y una tasa de transmisión de reserva para posibles fallas. Por tal razón, se propone que el empleo de los enlaces sea del $60 \%$ y que el BW de reserva dependa de las condiciones de la red de cada operador, así como también de las políticas de gestión que manejen. Dado que la utilización debe ser del $60 \%$, la capacidad requerida se obtiene multiplicando el espectro mínimo por una razón de 100 sobre el porcentaje de utilización de enlaces, más el ancho de banda de reserva, es decir [8]:

El BW de reserva expresado en la tabla anterior es impuesto por el operador, pero debe ser mínimo de un $20 \%$ de la capacidad total con el fin de garantizar calidad en el enlace.

La Fig. 11 muestra una aproximación lineal del espectro total requerido para el funcionamiento y soporte de la calidad de experiencia según el numero de abonados.

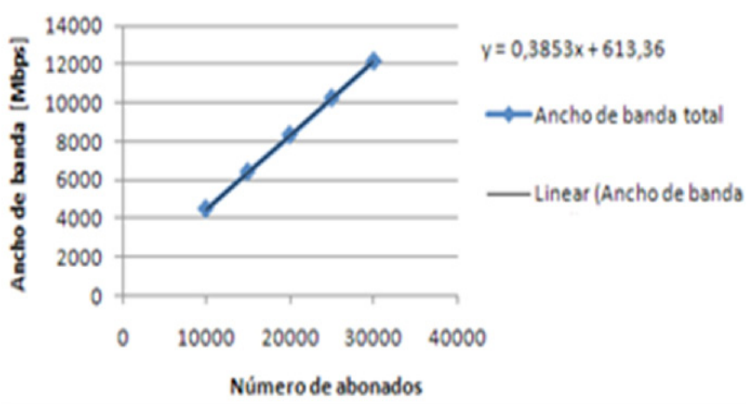

Fig. 11. Aproximación lineal de la capacidad requerida para soporte de IPTV.

La línea de color azul representa el ancho de banda total requerido según los datos de la tabla 2, y la recta de color negro es una aproximación matemática realizada por el software de Excel.

Teniendo en cuenta las consideraciones de dimensionamiento para el modelo de referencia propuesto en este trabajo, en la grafica anteriorse establece una aproximación matemática representada por la siguiente ecuación:

$$
y=0.385 x+613.36
$$

Donde la variable $Y$ representa la capacidad total requerida en Mbps, y $\mathrm{X}$ indica el número de abonados. De la Ec. (4) es posible 
deducir tres cosas muy importantes para tener en cuenta a la hora de diseñar una red para prestar el servicio de televisión por la red de datos; la primera es que la pendiente de 0.385 indica que por el momento el número de subscriptores de IPTV es muy bajo con relación al número de clientes potenciales que tiene el operador; la segunda es que si el número de terminales fuera cero existiría un mínimo ancho de banda de reserva en Mbps que es representado por la magnitud 613.36; por último que al ser una aproximación lineal se puede concluir que el BW requerido es directamente proporcional al número de clientes.

\section{IMPLEMENTACIÓN DE IPTV SOBRE IPV6 EN COLOMBIA}

\section{1 ¿Por qué IPTV sobre IPv6?}

El camino de la evolución tecnológica sugiere una actualización de IPTV, tanto en redes, tecnología y protocolos de transmisión; más aún cuando es un servicio en desarrollo y tan promisorio; por este motivo es necesario la implementación de estándares de última generación como el protocolo de Internet versión 6.

La televisión por la red de datos sobre IPv6, muestra mejoras al servicio si se compara con la utilización de IPv4, ya que es una solución a varias de las limitaciones que presenta esta versión. Las ventajas de IPTV6 son:

- Facilita, soporta y mejora la movilidad.

- Direccionamiento multicast a diferencia de broadcast, lo que facilita la implementación de PPV.

- Presta identificación de calidad de servicio y clase de servicio.

- Debido a que es un protocolo autoconfigurable, resuelve el escalamiento en el ruteo y hace que la adición de usuarios de Television sobre IP se realice con mayor facilidad.

- Mayor velocidad de transmisión gracias a la ampliación del tamaño del paquete que puede ser transportado sin fragmentación; lo que mejoraría la calidad de experiencia en servicios de VoD.

- Adecuado para nuevas aplicaciones como video juegos y demás funciones interactivas.

- Impulsa la subscripción de IPTV en todo tipo de dispositivos, debido a la gran cantidad de direcciones que tiene IPV6.

Es importante la generación e implementación de propuestas IPTV6 dado que, es un avance a la convergencia tecnológica, lo que lleva consigo reducción de costos y facilidades para los operadores y clientes.

\subsection{Ente regulatorio del servicio de IPTV en Colombia}

Ministerio de Tecnologías de la Información y las Comunicaciones da las pautas legales para la implementación de IPTV en Colombia. De la posición adoptada por este Ministerio y sus posteriores desarrollos regulatorios, es posible extraer un principio de obligatoria observancia si se quiere dotar de previsibilidad y coherencia al marco normativo bajo el que se rigen los servicios de comunicaciones en Colombia. Ese principio propone que si una comunicación se origina o termina en un usuario de la red de Internet, entonces se estará frente a un servicio de aquellos tipificados como de valor agregado, con independencia de la tecnología usada para su transmisión o recepción por parte del cliente. Con fundamento en ello, se soporta una conclusión fundamental que sostiene que la tecnología IPTV, es un servicio de valor agregado [3].

A efectos de actualizar el marco regulatorio y hacerlo armónico con los nuevos escenarios convergentes, se expidió el pasado 31 de julio de 2007 el Decreto 2870 "por medio del cual se adoptan medidas para facilitar la Convergencia de los Servicios y Redes en materia de Telecomunicaciones", el cuál retoma el concepto de servicios de valor agregado, establecido de tiempo atrás en el Decreto 1900 de 1990, y lo define para 
escenarios de convergencia tecnológica. [3]. El decreto tiene por objeto establecer un marco reglamentario que permita la convergencia en los servicios públicos de telecomunicaciones y en las redes de telecomunicaciones del Estado, asegurar el acceso y uso de las redes y servicios a todos los habitantes del territorio, así como promover la competencia entre los diferentes operadores [42].

\subsection{Consideracion de implementación}

La implementación de la televisión sobre IPv6 genera cambios que permiten una transmisión más eficiente, mayor flexibilidad para introducir nuevas opciones en el futuro y menos límites.

Gracias a las ventajas de IPTV6 es posible la eliminación de mecanismo y protocolos añadidos a IPv4 (NAT y DHCP), Proxys, enrutamiento triangular, utilización de direcciones privadas y no válidas en Internet, etc [9]. Lo que hace que el servicio de IPTV sea más confiable, rápido, seguro, accesible, escalable y de mejor calidad.

Para la implementación de IPTV sobre IPV6 es necesario implementar y mejorar las tecnologías de acceso y distribución local, algunas sugerencias son expuestas a continuación.

\subsubsection{Tecnologías de acceso y distribu- ción local}

La infraestructura necesaria para el acceso y distribución de IPTV6 debe suplir los siguientes requisitos para la prestación del servicio:

- Entregar diferentes canales de televisión de manera simultánea en la misma conexión de acceso.

- Capacidad de 2.1 Mbps por canal de definición estándar (SDTV) y 8 Mbps en canales de alta definición (HDTV).

- Soporte de diferentes tecnologías de distribución de red. En este caso se propone G-PON.

- Soporte de capacidad de canal para nuevos subscriptores, la cual debe estar por encima de 4 Gbps para servicio de televisión y debe ampliarse si se adiciona la conexión a Internet.

A continuación se plantea una topología para la implementación de IPTV6, que cuenta con soporte de IPv6 en todos los dispositivos de capa de red. El backbone es un modelo óptico que trabaja sobre SDH. Este modelo garantiza mayor simplicidad a nivel de administración gracias a la reducción de capas necesarias a ser utilizadas en un entorno óptico.

Al utilizar IPTV6 la señal que viaja por la red de datoses empaquetada de la forma que se muestra en la Fig. 12, en esta se observa como viaja un paquete de IPTV sobre una red óptica, en este caso SDH.

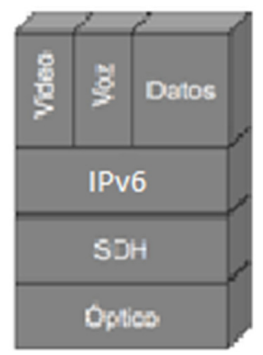

Fig. 12. Transporte del servicio de IPTV sobre SDH [41].

Además de la transformación de la señal mostrada en la grafica anterior, existen otras diferencias entre las redes según la versión del protocolo de Internet que utilice.

El esquema de la red de la Fig. 13 trabaja sobre IPv4 y el de la Fig. 14 sobre IPv6. 


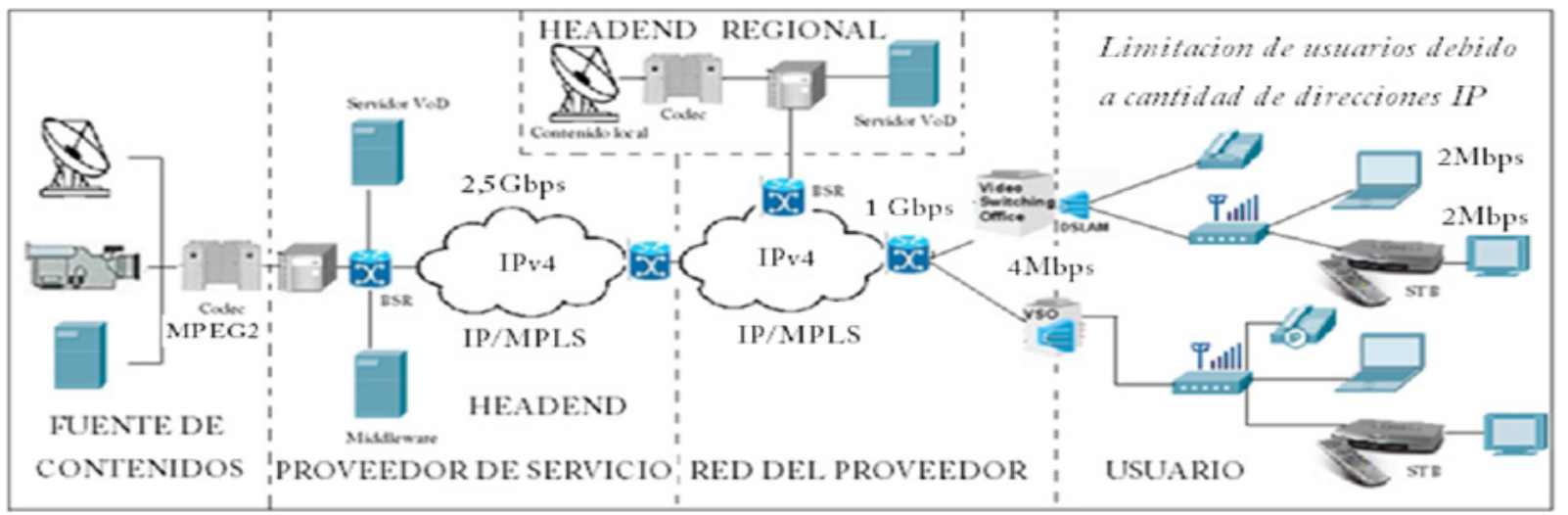

Fig. 13. Red IPTV sobre IPv4.

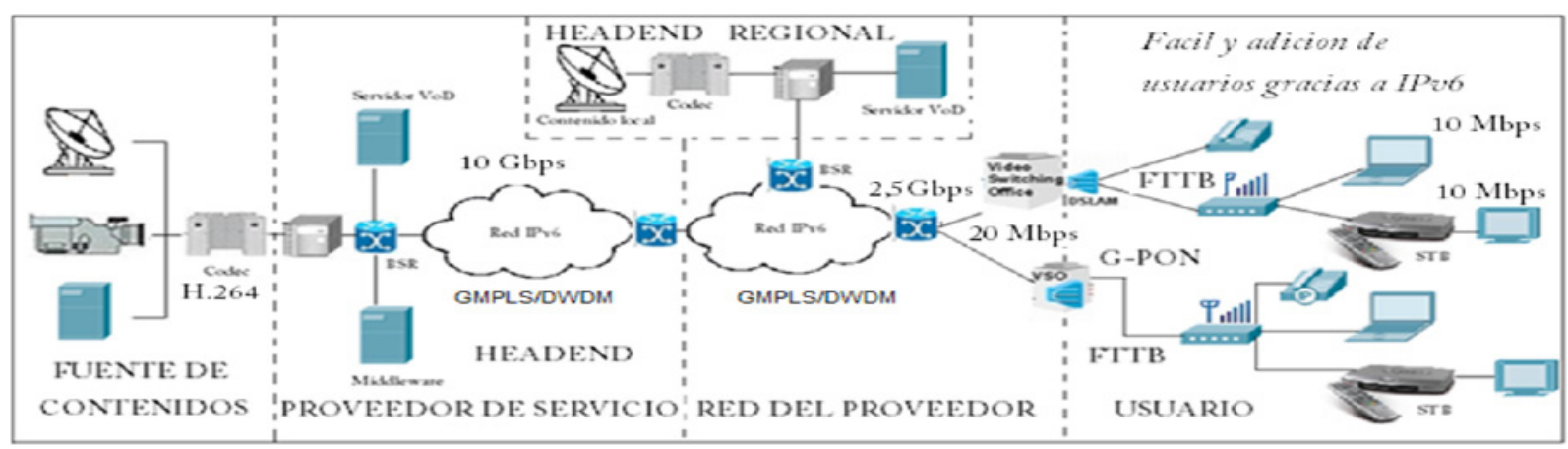

Fig. 14. Red propuesta para la implementación de IPTV sobre IPv6 en Colombia.

Las dos redes tienen la misma estructura, la cual esta dividida en 5 partes. Cada parte de la red IPVT6 presenta mejoras sobre la red esquematizada en IPv4, la cual es una representación de las topologías utilizadas actualmente por los ISP's en Colombia. Estas mejoras son:

- En el proveedor de contenidos se utiliza el formato de compresión H.264.

- El proveedor de servicio soporta GMPLS/DWDM para redes ópticas, lo que le permite obtener capacidades de canal muy superiores a las utilizadas actualmente en Colombia que son IPv4/ MPLS.

- La red del proveedor entrega al router de borde un BW superior, lo que permite incluir más usuarios y la mayor soporte para formatos HDTV.

- En la última milla se utiliza FTTB lo que garantiza tasas de transmisión alrededor de $20 \mathrm{Mbps}$ al usuario.

- Mayor cantidad de dispositivos con el servicio de IPTV gracias a las ventajas de IPv6 como movilidad y numero de direcciones IP.

A continuación se explicaran las ventajas de cada una de las partes que componen la red propuesta.

Proveedor de contenidos y Headend regional: hace la adquisición de algunas de las señales de IPTV y se encarga de codificar los contenidos en H.264 que reduce a la mitad los recursos de ancho de banda, obteniendo la misma calidad de video que MPEG2.

Proveedor de servicio: a diferencia de la red IPTV4, este se maneja mediante GM- 
PLS/DWDM el cual además de soportar conmutación de paquetes, soporta la conmutación en el tiempo, en longitud de onda y de fibras ópticas. Para ello, GMPLS extiende ciertas funciones base del tradicional MPLS y, en algunos casos, añade nueva funcionalidad. Adicionalmente soporta mayor cantidad de enlaces en paralelo, lo cual representa una ventaja para el proveedor de servicios. Por esta razón se evidencia un aumento en la capacidad de canal.

Red del proveedor: en el esquema propuesto, esta presenta las mismas ventajas que el proveedor de servicio.

Usuario: la implementación propuesta tiene un esquema típico de un usuario con servicio de "triple play", en comparación con una red sobre IPV4, se observa que en la red de última milla se implementa una infraestructura FTTB, esto con el fin de aumentar el ancho de banda, para este caso cambió de (4 a 20) Mbps. Adicionalmente, tiene la opción de incluir automáticamente VoIP.

La implementación de la topología propuesta en la Fig. 14, llevaría algún tiempo debido a la gran utilización de IPv4 y al poco uso hasta el momento de IPv6 en Colombia, aunque el ministerio encargado, ha propuesto la migración a IPv6 a partir de octubre de 2011. También seria una infraestructura de gran alto costo tanto para los proveedores de servicio como para los usuarios debido a la inversión a realizar para el mejoramiento de las tecnologías de acceso y distribución local.

\section{CONCLUSIONES}

IPTV es la tecnología que permite bidireccionamiento en el servicio de televisión, ya que admite la interacción entre el usuario y el proveedor del servicio, con este gran paso se transformara la manera en que se percibe la Televisión, además, porque también permite la conectividad a Internet y voz; todo esto converge en una misma red y no por separado como es en la actualidad. Aquí emerge la tecnología del futuro, que necesita una gran inversión debido a las limitaciones por el ancho de banda que esta maneja, pero en unos años será la tecnología que estará en cada hogar de Colombia.

IPTV es una tecnología que usa como soporte un servicio portador o telemático, representado por su red física, cableada o inalámbrica, para permitir el acceso a contenidos audiovisuales que se transportan por las redes de banda ancha; y a través de la cual se pueden prestar utilidades de valor agregado al usuario, haciendo uso de las facilidades del protocolo IP [3].

Sin importar como pueda ser asimilado el servicio de IPTV por parte del usuario, al utilizar dichas aplicaciones y de su posibilidad de acceso a contenidos audiovisuales, es claro que en el contexto de Internet, el uso de la tecnología IPTV no puede calificarse como operación del servicio de televisión, en ninguna de sus modalidades y por lo tanto en el caso de Co-lombia no puede ser regulado por el CNTV.

Dado que IPTV funciona sobre protocolos IPv4 e IPv6 que son protocolos de mejor esfuerzo más no confiables, es necesario adquirir políticas de calidad de servicio (Qos), que permitan el correcto funcionamiento de IPTV.

El secreto del mantenimiento y alta calidad del servicio IPTV cuando usa formatos de entrada como IP, SDI y RF, es un punto critico ya que compromete la integridad del sistema, por esto es esencial el monitoreo de la calidad del servicio antes de la inyección de las señales audiovisuales que son procesadas por el headend para salir a la red.

\section{AGRADECIMIENTOS}

Se destaca el agradecimiento a los docentes de la Universidad Distrital Francisco José de Caldas, el Ingeniero Danilo López Sarmiento y el Ingeniero Edmundo Vega, por 
el aporte de los materiales de consulta e investigación, además al Ingeniero Danilo

Referencias Bibliográficas

[1] Cullen Internacional. Cross-Country Analysis Western Eu-rope. IPTV Commercial Offers. Junio. 2006.

[2] A. Los Santos Aransay. Estado del arte en IPTV. Universidad de Vigo: multimedia e internet. Junio de 2009.

[3] Ministerio de Comunicaciones. Consulta pública: linea-mientos de política sectorial para el uso y aprovechamiento de la tecnología iptv. 2008.

[4] Home Electronic System (HES) gateway - part 1: a residential gateway model for HES. Information technology IEEE.

[5] Home Electronic System (HES) gateway - Part 1: A residen-tial gateway model for HES. Information technology. IEEE 1394.

[6] Triple-play Services Quality of Experience (QoE) Require-ments. DSL Forum Technical Report TR-126, 2007

[7] T. Muñoz. S. Muñoz. IPTV. Universidad del Cauca, 2010.

[8] F.Mieles, S. Torres, Consideraciones técnicas para la imple-mentación de tv digital sobre IP en Colombia. Universidad de los Andes, Colombia, 2010.

[9] IPV6 Colombia. Ministerio de Tecnologías de la Información y las Comunicaciones (TIC), [en línea]. Consultado en Febrero 3 de 2011, disponible en: http://184.106.30.252/E-DocumentManager/gallery/Documentos Acad $\%$ C3\%A9micos/ipv6word.pdf

[10] R. Ferro, C. Hernandez. Los sistemas IPVT: ¿una amenaza para los actuales medios de teledifusión? , 2010.

[11] J. Locatelli, S. Darin Estado del arte de IPTV en latinoameri-ca.Argentina, 2007.

[12] M. Rooney, Head of IPTV .Pace Micro Technology: IPTV White Paper, 2006.
Rodríguez y la Ingeniera Melissa Díaz por los aportes realizados.

[13] M. Azmi, R. Idrus, R. Budiarto, S. Ramadass. A proposed model for policybased routing rules in the ipv6 offering QoS for IPTV Broadcasting. NAv6 Centre, Universiti Sains Malay-sia, Pulau Pinang, Malaysia, 2008.

[14] C. Pantsios. IPTV: Televisión sobre IP, [en línea]. Consultado en Febrero 5 de 2011, disponible en: http://www. slideshare.net/RockyS11/iptv-televisin-sobre-ip

[15] [Grupo de expertos NGN.Cali. Redes NGN - Medición de la calidad des servicio. Colombia, 2008.

[16] Internet Protocol Television. For public television. Iowa, [en línea]. Consultado en Enero 4 de 2011, disponible en: http://www.answers.com/topic/ iptv\#ixzz1PaGQkhR4.

[17] M. Martínez. IPTV. Junio 2009. [en línea]. Consultado en Febrero 15 de 2011, disponible en: http://malorymartinez.blogspot.com/2009/06/quees-iptv.html

[18] J. Velásquez. Estudio de una red IP/ MPLS para agregar servi-cios de televisión IP en operadoras telefónicas fijas tradicionales para usuarios residenciales mediante tecnologías XDSL para la ciudad de Quito, Quito, 2010.

[19] Sistemas de Acceso Ópticos: estándares EPON/GPON,[en línea]. Consultado en Febrero 21 de 2011, disponible en: http://www.gatv.ssr.upm.es/stelradio/STEL/adjuntos/material_consulta/3_apuntes_gpon_y_epon.pd̄f.

[20] Digital Video Broadcasting; Transport of DVB service over IP. Julio 2004, [en línea]. Consultado en Diciembre 4 de 2010, disponible en: http://broadcasting.ru/pdf-standard-specifications/ internet $\% 20$ protocol/a086.tm3022r1. sb1338r1.tm-ipi10227r07.ipi\%20han- 
dbook\%20text.pdf

[21] Estudio Técnico sobre la Implementación de IPTV en la Red de ETAPA. [en línea]. Consultado en Febrero 3 de 2011, dis-ponible en: http://www.dspace.ups.edu.ec/bitstream/123456789/160/3/Capitu$10 \% 202$.pdf.

[22] Sistemas de Televisión sobre InfraestructuraIP: IPTV.Plataformas IPTV, [en línea]. Consultado en Febrero 3 de 2011, disponible en: http:// www.academy.itu.int/index.php/component/k2/item/download/662.

[23] J. She, F. Hou, P. Ho, L. Xie.IPTV over WiMAX: Key Success Factors, Challenges, and Solutions. IEEE Communications Magazine, 2007.

[24] RFC 1112: IGMPv1,[en línea]. Consultado en Febrero 13 de 2011, disponible en: ftp://ftp.rfc-editor.org/innotes/rfc1112.txt.

[25] RFC 2236: IGMPv2, [en línea]. Consultado en Febrero 6 de 2011, disponible en: ftp://ftp.rfc-editor.org/innotes/ rfc2236.txt

[26] RFC 3376: IGMPv3, [en línea]. Consultado en Febrero 1 de 2011, disponible en: ftp://ftp.rfc-editor.org/innotes/ rfc3376.txt

[27] C. Moreno, L. Fernández, Modelaje de protocolos de mem-brecía de grupos IP Multicast en redes Ethernet conmutadas. Caso de estudio: utilización de la videoconferencia, 2008

[28] G. O'Driscoll. Next Generation IPTV Services and Technolo-gies. 2008.

[29] Redes Ópticas Pasivas PON. Amalric. 2009, [en línea]. Consultado en Diciembre 12 de 2010, disponible en: http://es.debugmodeon.com/articulo/ redes-opticas-pasivas-pon.

[30] PON - Passive Optical Network, [en línea]. Consultado en Diciembre 12 de 2010, disponible en: http://www.infocellar.com/networks/new-tech/PON/ PON-real.htm.

[31] J. Guevara. Tecnologías de Redes Pon,[en línea]. Consultado en Febrero 7 de 2011, disponible en: http:// www.todotecnologia.net/wp-con-tent/ uploads/2010/06/Definicion_caracteristicas PON APOn BPON GE-

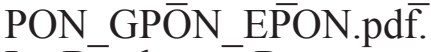

[32] L. Barahona. Resumen de redes de acceso por fibra óptica. Quito. Julio 2010, [en línea]. Consultado en Diciembre 12 de 2010 disponible en: http://es.scribd.com/doc/34376247/ REDES-PON-Redes-Opticas-Pasivas.

[33] R. Millán. GPON (Gigabit Passive Optical Network). 2007. [en línea], Consultado en Febrero 10 de 2011, disponible en: http://www.ramonmillan.com/tutoriales/gpon.php

[34] M. López, E. Moschim, F. Rudge. Estudio comparativo de redes Gpon y Epon. Mayo de 2009.

[35] DSL. Secretaría de Estado de Telecomunicaciones y para la Sociedad de la Información. España,[en línea]. Consultado en Febrero 21 de 2011, disponible en: ghttp://www.bandaancha. es/Informacion/Tecnologias/TecnologiasCableadas/Paginas/ADSL.aspx

[36] Quality of Service Network Performance Objectives for IP - Bases Service, ITU-T Rec, Y 1541, 2003.

[37] J. Amatriain, IPTV. Protocolos empleados y QoS, 2007

[38] "Quality of Service Network Performance Objectives for IP - Bases Service", ITU-T Rec, Y 1541, 2003.

[39] C. Viloria, J Freja, Y. Donoso, Análisis de rendimiento de la transmisión de IPTV sobre ADSL, WiFi y LAN Extended, 2008.

[40] F. Moumtadi, M. Escobar, R. López y S. Lande-ros.Reducción del retardo en el cambio de canal en servicios IPTV,2008.

[41] Topologías en redes SDH. [en línea]. Consultado en Febrero 20 de 2011, disponible en: https://www. tlm.unavarra.es/ daniel/docencia/ rba/rba09_10/slides/25-TopologiasYTransporteSDH.pdf.

[42] Ministerio de Comunicaciones. Decreto 2870 DE 2007 Diario Oficial No. 46.706. 31 de julio de 2007. 